\title{
Energy-based devices for hemostasis in thyroid surgery
}

\author{
Aleksander Konturek ${ }^{1}$, Bartłomiej Szpyra ${ }^{2}$, Małgorzata Stopa-Barczyńska $^{1}$, Marcin Barczyński $^{1}$ \\ ${ }^{1}$ Department of Endocrine Surgery, Third Chair of General Surgery, Jagiellonian University Medical College, Krakow, Poland; ${ }^{2}$ Doctoral School of \\ Medical Sciences, Jagiellonian University Medical College, Krakow, Poland \\ Contributions: (I) Conception and design: A Konturek; (II) Administrative support: A Konturek; (III) Provision of study materials or patients: A \\ Konturek, M Barczynski; (IV) Collection and assembly of data: B Szpyra, M Stopa-Barczynska; (V) Data analysis and interpretation: All authors; (VI) \\ Manuscript writing: All the authors; (VII) Final approval of manuscript: All authors. \\ Correspondence to: Aleksander Konturek, MD, PhD. Department of Endocrine Surgery, Third Department of General Surgery, Jagiellonian \\ University Medical College, 37 Prądnicka Street, 31-202 Krakow, Poland. Email: okont@mp.pl.
}

\begin{abstract}
The evolution of operative techniques in thyroid surgery that has taken place over the past several decades would not have been possible if not for a sui generis revolution in surgery at the turn of the 19th and 20th centuries. The three most important events of the end of the 19th century, i.e., the introduction of anesthesia, the use of artery forceps and ligation of blood vessels, as well as prophylactic management of perioperative infections decidedly affected the improvement of therapeutic results of thyroid surgery. Surgical treatment of thyroid diseases is associated with the possibility of complications developing, of which bleeding is among the most serious ones, starting from the need for an immediate reoperation and ending at a considerable damage to functionally important structures: the laryngeal nerves and parathyroid glands. The consensus reached between the development of science and progress in modern technologies has provided the basis for introducing and popularizing minimally invasive procedures, including operations using the approach through natural body openings. Such an unprecedented development of surgical techniques would not be possible without devices for closing blood vessels. Given the mechanism of hemostasis, current vascular sealing devices can generally be divided into three groups: ultrasonic, bipolarradiofrequency and hybrid systems combining both energy modalities. While analyzing the innovative, advanced technology of all the energy-based devices, it should be stated that only if they are used in a safe manner, quality of life of patients with various thyroid conditions is improved. The employment of such devices fully confirms their usefulness; nevertheless, all the advantages should never release the surgeon from the obligation to appropriately and safely identify the surrounding structures and let him uncritically use the new device.
\end{abstract}

Keywords: Thyroidectomy; new energy based devices; hemostatic agents

Submitted Oct 15, 2019. Accepted for publication Oct 29, 2019.

doi: 10.21037 /gs.2019.10.17

View this article at: http://dx.doi.org/10.21037/gs.2019.10.17

The evolution of operative techniques in thyroid surgery that has taken place over the past several decades would not have been possible if not for a sui generis revolution in surgery at the turn of the 19th and 20th centuries. The three most important events of the end of the 19th century, i.e., the introduction of anesthesia, the use of artery forceps and ligation of blood vessels, as well as prophylactic management of perioperative infections decidedly affected the improvement of therapeutic results and drastically decreased mortality rates down to an unthinkable $1 \%$ of patients operated on due to various thyroid conditions. The foundations of pathophysiology presented by Emil Theodor Kocher and standardization of surgical techniques proposed by Frank Lahey, William Halstedt or Jan Mikulicz-Radecki consolidated the position of classic thyroidectomy as the most effective method of treating thyroid gland diseases. Over the decades, the technique has undergone some minor alterations only, but the last three decades have marked a 
dynamic development of new instruments that have exerted the uppermost effect on improving the old and developing new operational techniques. The development of advanced surgical methods combined with a strife for performing the procedure in the least possible invasive manner with respect to the organism of the patient have increased the need for instruments that would restrict the intraoperative trauma. Appropriate hemostasis, atraumatic tissue preparation combined with protection against undue thermal injury that leads to damaging their structure are at present the foundations of modern surgery $(1,2)$.

Surgical treatment of thyroid diseases is associated with the possibility of complications developing, of which bleeding is among the most serious ones, starting from the need for an immediate reoperation and ending at a considerable damage to functionally important structures: the laryngeal nerves and parathyroid glands. Such complications most frequently were and still continue to be the result of improper identification of the tissues that surround the thyroid, as well as of lack of attentiveness to preparing them in an atraumatic manner. At present, the proper and careful surgical technique combined with attention paid to full identification of anatomical structures markedly decrease the number of complications. The consensus reached between the development of science and progress in modern technologies has provided the basis for introducing and popularizing minimally invasive procedures, including operations using the approach through natural body openings. Such an unprecedented development of surgical techniques would not be possible without devices for closing blood vessels. The aforementioned energy-based devices, using various energy types, have become increasingly more often employed; the devices combine various properties of other instruments, starting with preparation and ending with safe closure of blood vessels. The conclusions drawn from their use also describe other advantages, such as shortening the operational time, decreasing intraoperative blood loss, limiting the extent of the procedure, at the same time providing the above mentioned complete vascular hemostasis (scar length), or various degrees of an always genuine decrease in hospitalization costs and improvement in the quality of histological preparations to be evaluated and-by the same token—of the final diagnosis achieved through reduction of mechanical and thermal destruction of the thyroid tissues. Improvement has been also noted in the histopathological evaluation of the surgical margin of the resected tissue. A higher degree of mechanical and thermal destruction of the cells, leading to an irreversible damage of their structure, oftentimes rendered appropriate evaluation of the preparation impossible (3-5).

Thus, such devices have replaced classic blood vessel ligation and have become indispensable in endoscopic surgery, including low invasive procedures. Are there, then, any limitations in their use and is there a critical assessment of their employment in thyroid surgery?

In the group of complications following thyroid procedures, apart from life-threatening hemorrhage following goiter surgery, the second most common complication is injury of the recurrent laryngeal nerves. Side by side with injuries resulting from traction and maintaining tension of the surrounding tissues, thermal injuries are the most common. Dissociated in time, difficult to assess in the course of the very procedure, they pose a genuine and serious danger in thyroid surgery not only when performed by less experienced surgeons; such complications are also noted in specialized centers $(6,7)$.

Given the mechanism of hemostasis, current vascular sealing devices can generally be divided into three groups:

* ultrasonic systems (frequency 55 thousand $\mathrm{Hz}$ );

* electrothermal bipolar systems operating on radiofrequencies (from 2-4 MHz);

* hybrid systems combining the above-categorized energy modalities.

\section{Ultrasonic shears-The Harmonic Focus (HF) (Ethicon, Johnson and Johnson, Cincinnati, OH, USA)}

The ultrasonic shears are a low-temperature device operating within the range of 100 to $150{ }^{\circ} \mathrm{C}$. The effect of the shears exerted upon the tissue may be divided into three periods that make use of three different physical-chemical phenomena. Hemostasis is achieved thanks to consecutively happening processes of coaptation, cavitation and coagulation as the temperature curve is increasing within the afore-mentioned range. The process is initiated by the generator that-acting through the piezoelectric converter-emits vibrations with the frequency of more than 55 thousand Hz. At the junction of the tissue and the working tip, with the temperature reaching $50-63{ }^{\circ} \mathrm{C}$, the tertiary structure hydrogen bonds break and the so-called "peptide glue" appears in the intercellular space; the glue stops bleeding closing the capillary vessels (coaptation). Subsequently, when the temperature reaches $100{ }^{\circ} \mathrm{C}$, water evaporates from the tissues what causes "jostling" of the dissected surfaces at both sides of the "edge". 
Thus, a phenomenon occurs that is termed the "cavity effect" (cavitation). In the last stage, when the temperature reaches $100-150{ }^{\circ} \mathrm{C}$, blood vessels with the diameter of up to $5 \mathrm{~mm}$ close (coagulation). The advantage of the device is no electric current flowing through the body of the patient as well as a low degree of marginal and deep traumatization of the tissue (the marginal necrosis of 1-2 $\mathrm{mm}$ along the dissection line at electrocoagulation of $4-6 \mathrm{~mm}$ in all directions). Numerous publications on the use of the ultrasonic shears in thyroid surgery present conclusions addressing such advantages as decreasing the operational time, decreasing postoperative drainage, limiting pain and reducing the length of the dissection line. No significant differences have been noted in the rate of possible complications manifested as the recurrent laryngeal nerve paralysis and hypoparathyroidism, either permanent or transient. Nevertheless, only observations made and based on animal models have provided reliable information on the effect of temperature on the functioning of the recurrent laryngeal nerves and parathyroid glands. In case of the ultrasonic shears, the safe distance should be greater than $1 \mathrm{~mm}$ and the cooling time of the tip prior to its repeated activation should not be shorter than 10 seconds when the "cooling" occurs at room temperature and 2 seconds in case the tip is placed on the surface of the sternocleidomastoid muscle $(5,8-11)$.

\section{LigaSureTM Small Jaw (LSJ) (Medtronic, produced by Covidien, Minneapolis, MN, USA)}

Energy devices based on radio frequency cause denaturation of collagen and elastin fibers, while closing the tips triggers a sui generis effect of a network creation producing a new structure of tissue composed of collagen and elastin. Operating according to an appropriate algorithm, energy platforms determine the optimal time and amount of the supplied energy, while the reaction system controlled by the feedback mechanism (the so-called TissueFect ${ }^{\mathrm{TM}}$ detection technology) automatically strops energy supply after the cycle is completed. The system operates within the range of $3,333 \mathrm{~Hz}$, appropriately adjusting the initial power to achieve the desired effect via a proper amount of energy. Thanks to the technique we are able to close vessels as small as $7 \mathrm{~mm}$ in diameter and the time when the power supply is paused affects cooling of the working tip. The presented characterization of the device is reflected by the lesser degree of lateral heat dispersion and by the same token in limiting the effect of temperature upon the tissue functioning. The average temperature of the working tip after 5 seconds of its operation should not exceed $44{ }^{\circ} \mathrm{C}$. Nevertheless, the temperature may increase during the active operation of the device up to $96{ }^{\circ} \mathrm{C}$, with the average value of approximately $60{ }^{\circ} \mathrm{C}$. The disadvantage of the devices used to be lack of combination of simultaneous coagulation and the function of tissue dissection. The problem was solved by using a small cutting edge at the tip of the device. Animal studies allowed for standardizing the technique, at the same time providing information on the effect of high temperatures on conduction in the peripheral nerves. The critical temperature for their functioning has been accepted to be $60^{\circ} \mathrm{C}$, hence the recommended distance of the LigaSureTM device from the nerves is more than $2 \mathrm{~mm}(>2 \mathrm{~mm})$ with the tip being cooled for more than 2 seconds at room temperature or with a simultaneous placing on the adjacent muscle (the sternocleidomastoid muscle) in case of a repeated activation $(8,12,13)$.

One of the variants of the devices based on high frequency radio waves operation are (RadioSURG ${ }^{\circledR}$ 2,2 and ENSEAL, Ethicon Endo-Surgery) devices that operate using the frequency of 2,2 $\mathrm{MHz}(2,200,000$ cycles per second). The energy concentrated at the ends of the operating bipolar electrodes causes rapid movement of particles, triggering heat production and cell evaporation, while shrinkage of the collagen fibers additionally evokes the effect of local hemostasis. And thus, the tissue temperature is within the range of $60-100{ }^{\circ} \mathrm{C}$, while the tip of the device maintains a low temperature. In keeping with the data from the literature, employing the devices in thyroid surgery allows for shortening the operative time and cost reduction, at the same time making is possible to safely use the devices in surgery of vital structures (the recurrent laryngeal nerve and parathyroid glands) when the team is experienced. Unfortunately, there are no extensive, multicenter studies what may affect the interpretation of the results and limit the use of the method $(14,15)$.

\section{Thunderbeat (TB) (Olympus, Japan)}

One of the most important hybrid systems that combine the ultrasound and bipolar modalities to close and dissect blood vessels with the diameter up to $7 \mathrm{~mm}$. The above devices, easy and ergonomic in both open and endoscopic thyroidectomies, have become highly popular in recent years. Numerous publications have confirmed their effectiveness in thyroid surgery; they reduce the operative time and in endoscopic surgery, they additionally improve visibility reducing the amount of 
Table 1 Characteristic of the major sealing systems using in the thyroid surgery

\begin{tabular}{|c|c|c|c|c|}
\hline & $\begin{array}{l}\text { The Harmonic Focus } \\
\text { (HF) }\end{array}$ & LigaSureTM Small Jaw (LSJ) & The Thunderbeat & $\begin{array}{l}\text { Bipolar radiofrequency } \\
\text { ablation device }\end{array}$ \\
\hline Kinds of energy & Ultrasound $55 \mathrm{kHz}$ & Bipolar 3.3 kHz & $\begin{array}{l}47 \mathrm{kHz} \text { combine the ultrasound } \\
\text { and bipolar modalities }\end{array}$ & $\begin{array}{l}\text { High radiofrequency } \\
\text { waves } 2.2 \mathrm{MHz}\end{array}$ \\
\hline Vessel sealing & $5 \mathrm{~mm}$ & $7 \mathrm{~mm}$ & $5-7 \mathrm{~mm}$ & No data \\
\hline Lateral thermal spread & $2-3 \mathrm{~mm}$ & $1 \mathrm{~mm}$ & $2-3 \mathrm{~mm}$ & $1 \mathrm{~mm}$ \\
\hline $\begin{array}{l}\text { Ergonomic of the hand } \\
\text { piece }\end{array}$ & Good & Moderate & Good & Moderate \\
\hline $\begin{array}{l}\text { Safety distance from } \\
\text { RLN and parathyroids }\end{array}$ & $2 \mathrm{~mm}$ & $>2 \mathrm{~mm}$ & $>3 \mathrm{~mm}$ & No data \\
\hline Cost & Moderate & Moderate-higher & Moderate & Lower \\
\hline
\end{tabular}

smoke. Animal studies have led to developing safety rules of dissecting the tissues in the vicinity of the recurrent laryngeal nerve, with the distance from the nerve being $>3 \mathrm{~mm}$ and the time of a single exposure lower than 8 seconds ( $<8$ seconds) (Table 1) (12,16-18).

\section{Conclusions}

From the viewpoint of the patient, the most important goal of all the therapeutic modalities, including in particular surgical treatment, is prompt recovery. Such a standpoint makes the operation one of the therapeutic stages only, while the possibility of complications developing should be minimized. The considerable progress observed in the past several score years in monitoring quality of life following surgical procedures involving the thyroid gland would not have been possible without development of modern operative techniques and improvement of the instruments. The introduction of the new generation of devices based on various types of energy and employed in closing and dissecting the blood vessels has provided opportunities for performing procedures in a less invasive manner, with a minimal intraoperative blood loss, shortened operational and hospitalization time. Unfortunately, along with the introduction of the energy-based devices, possible causes of complications have also changed; among them, an intraoperative injury of the recurrent laryngeal nerve has been and continues to be the result of non-adherence to the appropriate surgical technique, leading in consequence to dysfunction of nerve conductivity with the anatomical integrity of the nerve being preserved. At present, the second most common cause of nerve injuries resulting from the mechanism of traction (71\%), is nothing else but the effect of high temperatures in the vicinity of the nerve, causing is thermal damage. In turn, the introduction of intraoperative recurrent laryngeal nerve neuromonitoring has fundamentally affected the quality improvement, but at the same time has provided an excellent tool for assessing the correctness of the performed surgical procedures while using devices for vessel closure in experimental studies, including animal models. Thanks to such studies, it has been possible to formulate a new classification system of the degree of intensity and possibility of returning of the function of the injured nerves depending on the mechanism of the damage. Dionigi et al. proposed that thermal injuries (Class-D) were defined as injuries of moderate or high severity, with a slower recovery time $(91 \pm 11$ days), higher rate of permanent RLN palsy (28\%), physical changes (22\%). This type of RLN is much more dangerous for the nerves at risk (12,18-20).

And thus, with the introduction of energy-based devices in thyroid surgery, the surgical principles have been considerable changed, so that the knowledge of anatomy and physiology be supplemented by the knowledge of the 
below specified subjects:

* the type of vessel-closing devices;

* the mechanism of their operation;

* the safe distance from life-important structures;

* the activation time and safe latency before the device is employed again;

* employment of such devices by highly-experienced surgeons following prior theoretical and practical preparation.

Studies on animal models have provided not only functional, but also anatomopathological information on the effect of high temperature on the degree of nerve injury, demonstrating that infringement of the endoneurium most often results in permanent paralysis of the vocal folds. Hemostasis, being the foundation of each surgical procedure, is a "sine qua non" condition of thyroid surgery. The limited operational field, rich vasculature and the vicinity of life-important anatomical structures cause even mild bleeding to blur the proper picture of the surgical area.

Summing up, it should be stated that the progressive development of instruments allows for a wide access to energy-based devices; nevertheless, their employment should be based on defined principles. Below, the reader will find some of such rules:

* Detailed visualization of the nerve course prior to activation of the device;

* Knowledge of the safe distance from the nerve; the recommended distance should not be lower than $2-3 \mathrm{~mm}$;

* Prohibition of the tip of the device contacting the nerve immediately after the device activation;

* The minimum time needed for a repeated activation differs for particular devices from 2 to 10 seconds when the tip is free and at the ambient temperature and 2 seconds after touching the adjacent muscle structures;

* The lateral heat wave dissipation from 1.5 to $3.3 \mathrm{~mm}$ beyond the region of the end of the working tip of the device.

While analyzing the innovative, advanced technology of all the energy-based devices, it should be stated that only if they are used in a safe manner, quality of life of patients with various thyroid conditions is improved. The employment of such devices fully confirms their usefulness; nevertheless, all the above discussed advantages should never release the surgeon from the obligation to appropriately and safely identify the surrounding structures and let him uncritically use the new device (19-22).

\section{Acknowledgments}

None.

\section{Footnote}

Conflicts of Interest: The authors have no conflicts of interest to declare.

Ethical Statement: The authors are accountable for all aspects of the work in ensuring that questions related to the accuracy or integrity of any part of the work are appropriately investigated and resolved.

\section{References}

1. Dorairajan N, Pradeep PV. Vignette thyroid surgery: a glimpse into its history. Int Surg 2013;98:70-5.

2. Harvey SC. The History of Hemostasis. BJS 1929;17:348.

3. Ortega J, Sala C, Flor B, et al. Efficacy and costeffectiveness of the UltraCision harmonic scalpel in thyroid surgery: an analysis of 200 cases in a randomized trial. J Laparoendosc Adv Surg Tech A 2004;14:9-12.

4. Cordón C, Fajardo R, Ramirez J, et al. A randomized, prospective, parallel group study comparing the harmonic scalpel to electrocautery in thyroidectomy. Surgery 2005;137:337-41.

5. Barczyński M, Konturek A, Cichoń S. Minimally invasive video-assisted thyreoidectomy (MIVAT) with and without use of harmonic scalpel--a randomized study. Langenbecks Arch Surg 2008;393:647-54.

6. Lang BH, Ng SH, Lau LL, et al. A systematic review and meta-analysis comparing the efficacy and surgical outcomes of total thyroidectomy between harmonic scalpel versus ligasure. Ann Surg Oncol 2013;20:1918-26.

7. Ecker T, Carvalho AL, Choe JH, et al. Hemostasis in thyroid surgery: harmonic scalpel versus other techniques--a meta-analysis. Otolaryngol Head Neck Surg 2010;143:17-25.

8. Dionigi G, Boni L, Rausei S, et al. The safety of energybased devices in open thyroidectomy: a prospective, randomised study comparing the LigaSure ${ }^{\mathrm{TM}}$ (LF1212) and the Harmonic® FOCUS. Langenbecks Arch Surg 2012;397:817-23.

9. Dionigi G, Van Slycke S, Rausei S, et al. Parathyroid function after open thyroidectomy: A prospective randomized study for ligasure precise versus harmonic FOCUS. Head Neck 2013;35:562-7. 
10. Cosenza G, Morano C, Cilurso F, et al. LigaSure Small Jaw vs. Harmonic Focus and Clamp-and- Tie Technique in Total Thyroidectomy for Benign Disease: A Prospective Randomized Trial. Clin Surg 2018;3:2038.

11. Zhang L, Li N, Yang X, et al. A meta-analysis comparing the outcomes of LigaSure Small Jaw versus clamp-and-tie technique or Harmonic Focus Scalpel in thyroidectomy. Medicine (Baltimore) 2017;96:e6141.

12. Dionigi G, Wu CW, Kim HY, et al. Safety of energy based devices for hemostasis in thyroid surgery. Gland Surg 2016;5:490-4.

13. Hammad AY, Deniwar A, Al-Qurayshi Z, et al. Prospective Study Comparing the Efficacy and Surgical Outcomes of Harmonic Focus Scalpel Versus LigaSure Small Jaw in Thyroid and Parathyroid Surgery. Surg Innov 2016;23:486-9.

14. Baldwin $K$, Haniff $M$, Somasundar P. Initial experience using a bipolar radiofrequency ablation device for hemostasis during thyroidectomy. Head Neck 2013;35:118-22.

15. Kadem SG, Alabbood MH. Safety and Efficacy of Bipolar Radiofrequency Ablation Device in Hemostasis during Thyroidectomy in Comparison with Ultrasonic Scalpel: A Comparative Study. Indian J Endocrinol Metab 2019;23:76-80.

Cite this article as: Konturek A, Szpyra B, Stopa-Barczyńska M, Barczyński M. Energy-based devices for hemostasis in thyroid surgery. Gland Surg 2020;9(Suppl 2):S153-S158. doi: 10.21037/ gs.2019.10.17
16. Tamura A, Tsutsui H, Yano Y, et al. Thermal effects of the Thunderbeat ${ }^{\mathrm{TM}}$ device on the recurrent laryngeal nerve during thyroid surgery. Surg Today 2018;48:1076-80.

17. Materazzi G, Ambrosini CE, Fregoli L, et al. Prevention and management of bleeding in thyroid surgery. Gland Surg 2017;6:510-5.

18. Dionigi G, Chiang FY, Kim HY, et al. Safety of LigaSure in recurrent laryngeal nerve dissection-porcine model using continuous monitoring. Laryngoscope 2017;127:1724-9.

19. Dionigi G, Wu CW, Kim HY, et al. Severity of recurrent laryngeal nerve injuries in thyroid surgery. World J Surg 2016;40:1373-81.

20. Wu CW, Randolph GW, Lu IC, et al. Intraoperative neural monitoring in thyroid surgery: lessons learned from animal studies. Gland Surg 2016;5:473-80.

21. Konturek A, Barczyński M, Stopa M. Total thyroidectomy for non-toxic multinodular goiter with versus without the use of harmonic FOCUS dissecting shears - a prospective randomized study. Wideochir Inne Tech Maloinwazyjne 2012;7:268-74.

22. Seehofer D, Mogl M, Boas-Knoop S, et al. Safety and efficacy of new integrated bipolar and ultrasonic scissors compared to conventional laparoscopic 5-mm sealing and cutting instruments. Surg Endosc 2012;26:2541-9. 